\title{
Electronic structure of the metallic oxide $\mathrm{ReO}_{3}$
}

\author{
J. Falke $\odot,{ }^{1}$ C. F. Chang $\odot,{ }^{1}$ C. E. Liu,${ }^{1,2}$ D. Takegami, ${ }^{1}$ A. Melendez-Sans $\odot,{ }^{1}$ C.-S. Chen $\odot,{ }^{1,2,{ }^{*}}$ L. Zhao,${ }^{1}$ \\ A. C. Komarek, ${ }^{1}$ C.-Y. Kuo, ${ }^{1,2,3}$ C. T. Chen, ${ }^{3}$ and L. H. Tjeng ${ }^{1}{ }^{1}$ \\ ${ }^{1}$ Max Planck Institute for Chemical Physics of Solids, Nöthnitzer Straße 40, 01187 Dresden, Germany \\ ${ }^{2}$ Department of Electrophysics, National Yang Ming Chiao Tung University (NYCU), 1001 University Road, Hsinchu 30010, Taiwan \\ ${ }^{3}$ National Synchrotron Radiation Research Center (NSRRC), 101 Hsin-Ann Road, Hsinchu 30076, Taiwan
}

(Received 16 November 2020; accepted 23 February 2021; published 15 March 2021)

\begin{abstract}
We have investigated the electronic structure of the metallic oxide $\mathrm{ReO}_{3}$ using bulk-sensitive angle-resolved soft-x-ray and angle-integrated hard-x-ray photoelectron spectroscopy. We observed clear dispersions of the $\operatorname{Re} 5 d$ - and $\mathrm{O} 2 p$-derived bands as well as the momentum splitting of the Fermi surface due to the Re $5 d$ spin-orbit interaction. We found that density-functional-based band structure methods can provide an accurate description of the observed electronic states. To achieve the accuracy it was necessary to utilize hybrid functionals for the calculations.
\end{abstract}

DOI: 10.1103/PhysRevB.103.115125

\section{INTRODUCTION}

Transition-metal oxides display a remarkably wide range of fascinating physical phenomena. These include metalinsulator and spin-state transitions, colossal magnetoresistance, superconductivity, and multiferroicity [1-4]. The oxide $\mathrm{ReO}_{3}$ is in this respect quite atypical: it is nonmagnetic [5], despite its $d$ shell being partially filled, and it is highly metallic. In fact, it has the highest conductivity of all oxides, comparable with that of copper or silver [6-9]. The temperature dependence of the resistivity is remarkably strong and signals the important role of optical lattice vibrations $[9,10]$.

$\mathrm{ReO}_{3}$ crystallizes in a structure composed of a threedimensional, regular network of corner-sharing $\mathrm{ReO}_{6}$ octahedra. Its undistorted cubic crystal structure with space group $P m \overline{3} m$ is closely related to the $\mathrm{ABO}_{3}$ perovskite structure but with the A-site remaining unoccupied, see Fig. 1(a). Due to this, the oxygen ions have a large amount of freedom lateral to their bonding axis, which manifests in an enhanced thermal displacement parameter and negative thermal expansion between $T=2 \mathrm{~K}$ and $200 \mathrm{~K}$ [11-15]. $\mathrm{ReO}_{3}$ undergoes multiple structural transitions at high pressures [16,17]. The material also readily absorbs hydrogen due to the vacant body-centered lattice site and finds application as a hydrogenation catalyst in the chemical industry $[18,19]$.

The electronic states of $\mathrm{ReO}_{3}$ were studied theoretically already early on using band structure calculations [20-25]. The results were compared favorably with findings from experiments using optical spectroscopy [26,27], de Haas van Alphen

\footnotetext{
*Present address: College of Electrical Engineering and Computer Science, National Taiwan University, Taipei 10617, Taiwan.

Published by the American Physical Society under the terms of the Creative Commons Attribution 4.0 International license. Further distribution of this work must maintain attribution to the author(s) and the published article's title, journal citation, and DOI. Open access publication funded by the Max Planck Society.
}

effect $[28,29]$, and $\mathrm{x}$-ray spectroscopies [30-34]. $\mathrm{ReO}_{3}$ was also compared to other oxides of the $3 d$ series having the same nominal $d^{1}$ configuration, and one can safely argue that $\mathrm{ReO}_{3}$ is not a strongly correlated system [35-37]. One may infer that this is closely related to the rather extended nature of the $\mathrm{Re}$ $5 d$ wave functions.

Despite the massive progress in electronic structure calculations and experimental methods in the last decades, it is somewhat surprising that to our knowledge there are no studies on the electronic structure of $\mathrm{ReO}_{3}$ using angle-resolved photoelectron spectroscopy (ARPES). The observation of dispersive bands will provide details not accessible from wave-vector $(k)$ integrated measurements. For example, information about the influence of the $5 d$ spin-orbit interaction on the Fermi surface can be gathered. Also the presence of possible self-interaction effects can be unveiled and quantified.

It is with the above-mentioned considerations that we have performed ARPES. Since $\mathrm{ReO}_{3}$ is three-dimensional, we have used soft $\mathrm{x}$ rays in order to cover a wide range of $k$ values in all three directions. It turned out that the probing depth of the photoemission experiment also needs to be maximized as to obtain spectra that are representative for the bulk material. To this end, the use of soft $\mathrm{x}$ rays for the ARPES and angle-integrated photoelectron spectroscopy (SXPES) is necessary. In addition, hard-x-ray photoelectron spectroscopy (HAXPES) for angle-integrated spectra was applied to verify the integrity of the spectra taken with soft $x$ rays. On the theoretical side, we need to make use of hybrid functionals in the band structure calculations in order to accurately reproduce the experimental spectra, demonstrating that self-interaction effects should not be neglected.

\section{METHODS}

Single crystals of $\mathrm{ReO}_{3}$ were grown by chemical vapor transport at the MPI CPfS Dresden and oriented using Laue diffraction. Soft x-ray ARPES and SXPES experiments were performed at the NSRRC-MPI TPS 45A Submicron Soft 


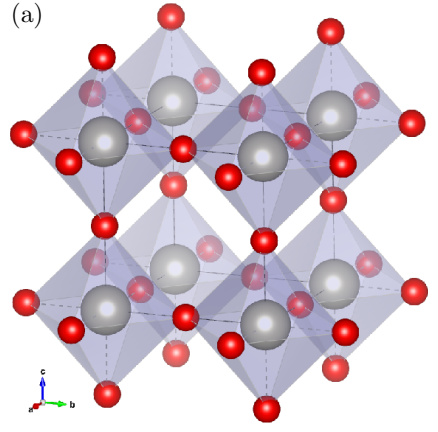

(b)

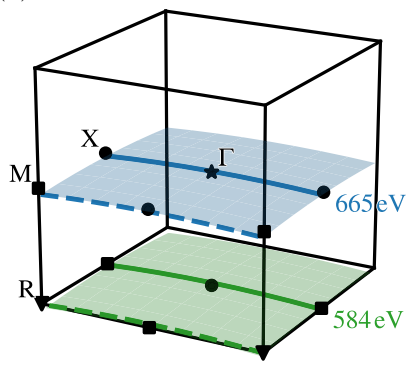

FIG. 1. (a) Crystal structure of $\mathrm{ReO}_{3}$. The Re sites (gray, large) are at the corners of the cubic unit cell and are octahedrally coordinated by six neighboring oxygens (red, small). The oxygens in turn are located on the edges of the unit cell and are thus linearly coordinated. This corresponds to the undistorted perovskite structure with a missing body-centered site. (b) Brillouin zone of $\mathrm{ReO}_{3}$ with high-symmetry points $\Gamma$ (star), X (circle), M (square), and R (triangle). Blue (green) shaded surface indicates $k$ space of states (at $E_{\mathrm{F}}$ ) reachable by $665 \mathrm{eV}(584 \mathrm{eV})$ photons. Blue and green lines indicate the trajectories of the ARPES spectra shown in this study.

X-ray Spectroscopy beamline [38] at the Taiwan Photon Source in Taiwan. The end station is equipped with an MB Scientific A-1 photoelectron analyzer. The overall energy resolution was set at $\approx 100 \mathrm{meV}$. The samples were cleaved in situ under ultrahigh vacuum conditions with a pressure better than $2.0 \times 10^{-10}$ mbar. Figure 2(a) shows the (100) surface of a sample after cleaving, and Fig. 2(b) the corresponding low-energy electron diffraction (LEED) pattern. The spectra were collected with the sample temperature kept at $40 \mathrm{~K}$.

HAXPES measurements were carried out at the MPINSRRC HAXPES end station [39] of the Taiwan undulator beamline BL12XU of SPring-8 in Japan. The photon energy was set to $h v=6.7 \mathrm{keV}$ and the overall energy resolution was $\approx 270 \mathrm{meV}$. The end station is equipped with two MB Scientific A-1 HE photoelectron analyzers. The photoelectrons were collected in the direction parallel to the electrical field vector of the photon beam with the sample temperature set at $80 \mathrm{~K}$.

The electronic structure calculations were performed using WIEN2K, an augmented plane wave plus local orbitals program [40,41]. Two kinds of parametrization of the exchangecorrelation potential were employed: the Perdew, Burke, and Ernzerhof (PBE) parametrization within the generalized gradient approximation (GGA) [42] and a screened hybrid functional for all the electrons [43-47]. The screened hybrid functional $E_{\mathrm{xc}}^{\text {hybrid }}$ was constructed such that a part $\alpha$ of the semilocal PBE-GGA exchange $E_{\mathrm{x}}^{\mathrm{GGA}}$ was replaced by the short-range part of the Hartree-Fock exchange $E_{\mathrm{x}}^{\mathrm{HF}}$ according to

$$
E_{\mathrm{xc}}^{\mathrm{hybrid}}=\alpha E_{\mathrm{x}}^{\mathrm{HF}}+(1-\alpha) E_{\mathrm{x}}^{\mathrm{GGA}}+E_{\mathrm{c}}^{\mathrm{GGA}},
$$

where $E_{\mathrm{c}}^{\mathrm{GGA}}$ is the correlation energy. For insulators and semiconductors, one may obtain readily accurate results by taking the inverse of the dielectric constant as an estimate for the $\alpha$ value [46], but for metals the situation is more complex [47]. Here we varied $\alpha$ from 0 to 0.3 , and the best fit was found for $\alpha=0.15$ as will be shown below. The Brillouin zone (a)

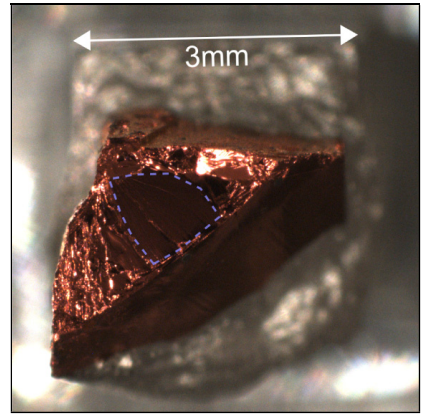

(b)

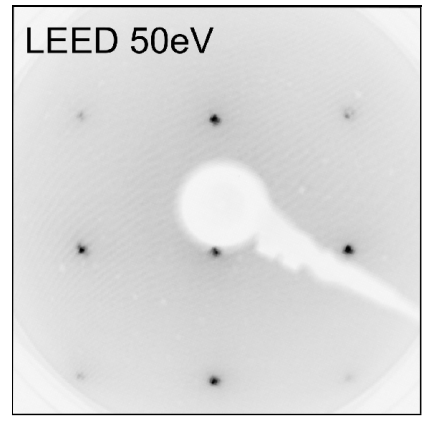

FIG. 2. (a) Photograph of cleaved (100) sample surface. The dashed line indicates the flat area used for the ARPES measurements. (b) Photograph of LEED pattern taken at room temperature of the sample surface after the ARPES measurement (total acceptance angle $\left.102^{\circ}\right)$.

was sampled by a well-converged regular $k$-point mesh with dimensions $16 \times 16 \times 16$ in the full zone. The Hartree-Fock exchange was calculated on an $8 \times 8 \times 8 k$-point submesh. All calculations were performed including relativistic spinorbit coupling unless otherwise indicated.

\section{RESULTS: HAXPES/SXPES AND DOS}

Figure 3 displays the calculated valence-band density of states (DOS) together with the experimental valence-band photoemission spectra. The GGA results in panel (a) reproduce the general features obtained in earlier calculations [20-25]: the $\operatorname{Re} 5 d$ and the $\mathrm{O} 2 p$ states contribute to the DOS in the energy range from -9 to $-3.7 \mathrm{eV}$ and from $-1.5 \mathrm{eV}$ and up. The DOS in the region between -3.7 and $-1.5 \mathrm{eV}$ consists mainly of the $\mathrm{O} 2 p$ states. The calculation also shows that the states closest to the Fermi level are made of Re $5 d t_{2 g}$ and $\mathrm{O} 2 p_{\pi}$ states, while states at the bottom of the valence band consist of $\operatorname{Re} 5 d e_{g}$ and $\mathrm{O} 2 p_{\sigma}$.

The valence band as measured by HAXPES, see panel (b), follows the calculated DOS quite well: the energies of the experimental peaks at $-9 \mathrm{eV},-6 \mathrm{eV}$, and $-3.5 \mathrm{eV}$ are close to the calculated ones, and the presence of a clear Fermi cutoff in the experiment is fully consistent with $\mathrm{ReO}_{3}$ being a good metal. One aspect to notice is that the intensity of the peak at $-3.5 \mathrm{eV}$ in the HAXPES is much lower than that of the $-2.9 \mathrm{eV}$ peak in the calculation. Looking at the photoionization cross-section tables [48-50], we find that for $h v=6.5 \mathrm{keV}$ the Re $5 d$ shell has more than two orders of magnitude larger cross section than the O $2 p$, meaning that the HAXPES experiment is essentially measuring mainly the Re $5 d$ partial DOS. To illustrate this aspect in a more quantitative manner, we have calculated the so-called cross-section weighted DOS, which consists of the sum of each partial DOS from Fig. 3(d) multiplied by the corresponding photoionization cross section, thereby taking into account also the specific geometry of the HAXPES experiment $[51,52]$ and by the Fermi function. The result as displayed by the dashed line in panel (b) thus indeed confirms that the DOS between -3.7 and $-1.5 \mathrm{eV}$ has an almost pure $\mathrm{O} 2 p$ character. 


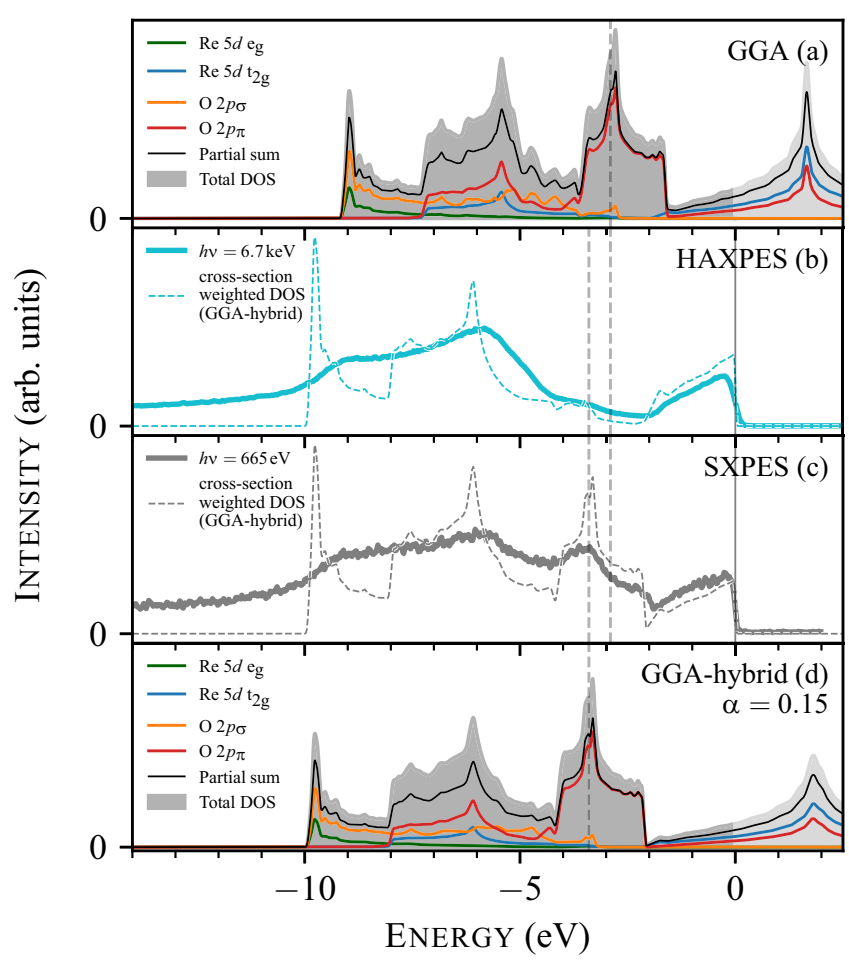

FIG. 3. Calculated density of states (DOS) and measured valence-band photoemission spectra. (a) Total and orbital projected DOS from the GGA calculation. (b) HAXPES spectrum $(h v=6.7$ $\mathrm{keV})$ and cross-section weighted DOS. (c) SXPES spectrum ( $h v=$ $665 \mathrm{eV}$ ) and cross-section weighted DOS. (d) Total and orbital projected DOS from the GGA calculation using a hybrid functional with $\alpha=0.15$. Dashed vertical line serves as a visual aid to highlight the shift in the O $2 p$ partial DOS due to the parameter $\alpha$.

Panel (c) of Fig. 3 shows the valence band measured using SXPES. The line shape is very similar to that of the HAXPES spectrum. The only significant difference is that the intensity of the $-3.5 \mathrm{eV}$ peak is much larger in the SXPES. This can be attributed to the fact that at $h v=665 \mathrm{eV}$ the photoionization cross section of the $\mathrm{O} 2 p$ is not small compared to that of Re $5 d[48,49]$. We have also calculated the cross-section weighted DOS for this photon energy, see the dashed line in panel (c). This result therefore provides another confirmation for the mainly $\mathrm{O} 2 p$ nature for the states located between -3.7 and $-1.5 \mathrm{eV}$. With HAXPES being a bulk sensitivity photoemission technique due to the high kinetic energy of the outgoing photoelectrons [53], the consistency between the SXPES and HAXPES spectra also implies that the use of soft $\mathrm{x}$ rays for valence-band photoemission provides sufficient probing depth as to obtain spectra that are representative for the bulk material. This is not an unimportant aspect, since for this particular case of $\mathrm{ReO}_{3}$ we noticed that if the kinetic energy of the photoelectrons is too low, e.g. below $150 \mathrm{eV}$, the spectra become distorted, see Appendix. This is presumably due to the presence of disorder or reconstructions at the sample surface, which in turn are likely related to the polar nature of the $\mathrm{ReO}_{3}$ (100) surface termination [54-59]. We therefore use soft $\mathrm{x}$ rays for our ARPES to ensure that the spectra provide information about the bulk electronic structure.
One striking difference between the calculated DOS shown in panel (a) and the experimental spectra in panels (b) and (c) of Fig. 3 is the positioning of the band which has an almost pure $\mathrm{O} 2 p$ character. The calculations place it between -3.7 and $-1.5 \mathrm{eV}$ with a peak at $-2.9 \mathrm{eV}$, while the experiment reveals the peak at $-3.4 \mathrm{eV}$. There is thus a discrepancy of about $0.5 \mathrm{eV}$. Similar misalignment of the $\mathrm{O} 2 p$ between calculation and experiment has also been observed in, for example, $\mathrm{Cu}_{2} \mathrm{O}[60,61], \mathrm{Ag}_{2} \mathrm{O}$ [62], and $\mathrm{NbO}$ [63], and can be taken as an indication for spurious self-interaction effects not sufficiently accounted for in conventional density-functional theory. To account for this, additional calculations were performed with a hybrid functional, where an admixture of the exact Hartree-Fock exchange to conventional density functional theory (DFT) functionals is incorporated [43-47]. We varied the mixing parameter $\alpha$ from 0 to 0.30 and found that $\alpha=0.15$ provides the best match between the calculation and the experiment. The resulting total DOS and partial Re $5 d$ and $\mathrm{O} 2 p$ DOS are plotted in panel (d) of Fig. 3. The experimental peak at $-3.4 \mathrm{eV}$ is now reproduced by the calculation. For $\mathrm{ReO}_{3}$, admixture of the Hartree-Fock exchange has the effect of rigidly shifting the primarily $\mathrm{O} 2 p$ derived bands to higher binding energy, while the Re $5 d$ bands around the Fermi level remain largely unaffected. The hybrid-functional approach thus provides a highly accurate description of the valence-band spectrum, with perhaps still some deviations for the bottom part of the valence band where a smaller $\alpha$ appears to be a better choice. With the Re $5 d$ states close to the Fermi level being of $t_{2 g}$ origin and those at the bottom of the valence band of $e_{g}$ type, we probably see here yet another example for the need to consider the orbital dependence of the exchange-correlation functional [47].

\section{RESULTS: ARPES, BANDS, AND FERMI SURFACE}

The ARPES measurements were performed on the (100) surface. To determine the inner potential $V_{0}$, a series of spectra were acquired at normal emission with systematic variation of the photon energy. A value $V_{0} \approx 4 \mathrm{eV}$ was determined for the inner potential relative to the Fermi level of $\mathrm{ReO}_{3}$. Figure 4 shows the ARPES results. The spectra in panels (a) and (b) were taken with $h v=665 \mathrm{eV}$ photons and those in panels (c) and (d) with $584 \mathrm{eV}$. Measurements shown in panels (a) and (c) were performed at emission angles close to the surface normal while those in panels (b) and (d) at larger emission angles. The trajectory of each spectrum within the Brillouin zone is also indicated in Fig. 1(b). In comparing the dispersive features in Figs. 4(b) and 4(c), we can observe that they are identical despite the spectra having been acquired at very different photon energies and different emission angles. This demonstrates the equivalence of the trajectories and consequently gives us confidence that our $V_{0}$ determination is accurate. In collecting and plotting the spectra, the nonnegligible transferred photon momentum carried by the soft $\mathrm{x}$ rays was taken into account.

The experimental ARPES results in Fig. 4 are overlaid with the bands of the GGA-hybrid calculations. The orbital characters are also indicated. We can observe that in all panels (a)-(d) there is excellent agreement between the bands from the experiment and those from theory. We now have a highly 

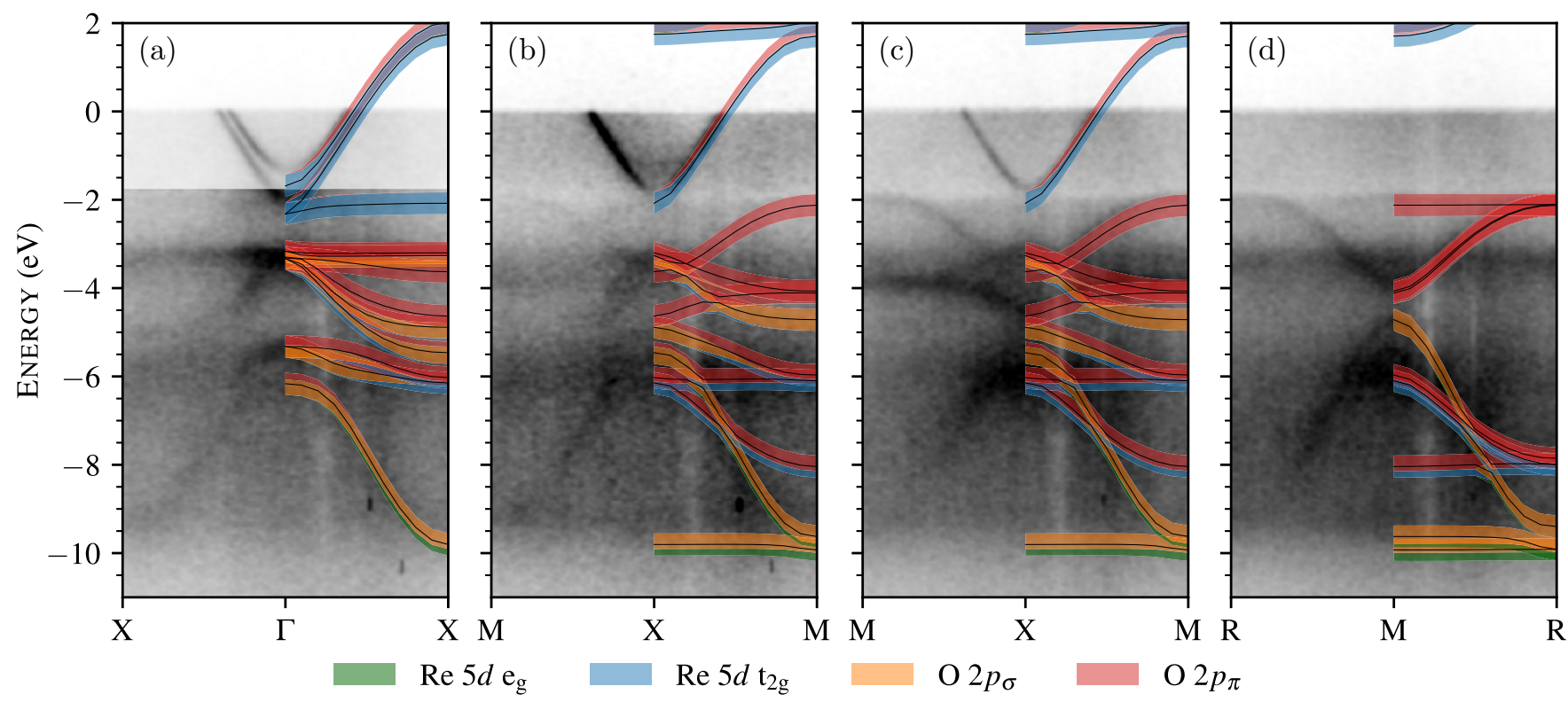

FIG. 4. ARPES spectra for different high-symmetry cuts of the Brillouin zone compared to the GGA-hybrid calculations. The photon energy in (a) and (b) is $h v=665 \mathrm{eV}$, and in (c) and (d) $h v=584 \mathrm{eV}$. The trajectories in the Brillouin zone of the spectra are also indicated in Fig. 1(b). Note: The intensity of the spectrum below $-1.8 \mathrm{eV}$ in (a) has been multiplied by a factor of 3 to enhance the visibility of the lower bands. The thickness of the colors indicates the relative magnitude of projected orbital characters.

detailed look at the electronic states and we can infer that use of the hybrid functional with $\alpha=0.15$, as determined from the angle-integrated spectra, provides very accurate results.

An important detail which should be noticed is that in panel (a) of Fig. 4 the bands closest to the Fermi level are split. These are $\operatorname{Re} 5 d t_{2 g}$ derived states, and they are split by the spin-orbit interaction for $k$ going from $\Gamma$ to $\mathrm{X}$ [20]. The energy splitting at $\Gamma$ is approximately $450 \mathrm{meV}$. Band structure calculations that do not include the spin-orbit interaction do not show such a splitting $[20,24,25]$.

From the ARPES data we can also obtain information about the Fermi surface. Figure 5 plots the Fermi-level intensities of spectra having $k$ wave vectors in the $\Gamma-\mathrm{X}-\mathrm{M}$ plane. The data were obtained with $h v=664.8 \mathrm{eV}$. Three sheets are

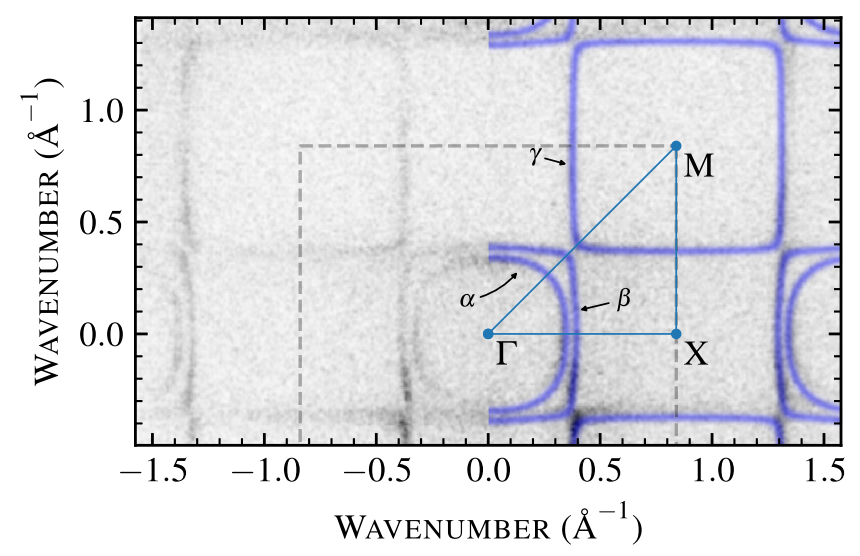

FIG. 5. Fermi surface cut in the $\Gamma-\mathrm{X}-\mathrm{M}$ plane. The experimental data were taken with $h v=664.8 \mathrm{eV}$, and the band structure results are from the GGA-hybrid calculations. Brillouin zone boundary is marked with the dashed line. visible: the $\alpha$ and $\beta$ sheets centered around the $\Gamma$ point, and the $\gamma$ sheet centered around the M point. We have also made an overlay with the Fermi surface from our band structure calculations using the GGA-hybrid functional. The match between theory and experiment is excellent.

Our next step is to investigate how the Fermi surface of $\mathrm{ReO}_{3}$ depends on corrections for self-interaction effects and on the Re $5 d$ spin-orbit interaction. To this end we compare the Fermi surface calculated with the GGA-hybrid functional with that of the standard GGA and that of the GGA without the spin-orbit interaction. The results are displayed in Fig. 6. We can observe that the Fermi surface of the GGA hybrid is practically identical with that of the GGA, see panels (a) and (b). This is perhaps somewhat surprising since the states that form the Fermi surface are made of the hybridized Re $5 d t_{2 g}$ and the $\mathrm{O} 2 p_{\pi}$, see Fig. 3 panel (a), and one may then expect that modifying the energy of the $\mathrm{O} 2 p_{\pi}$ when using the hybrid functional, see Fig. 3 panel (d), will modify the states near the Fermi level. Apparently, these changes are minor and can be

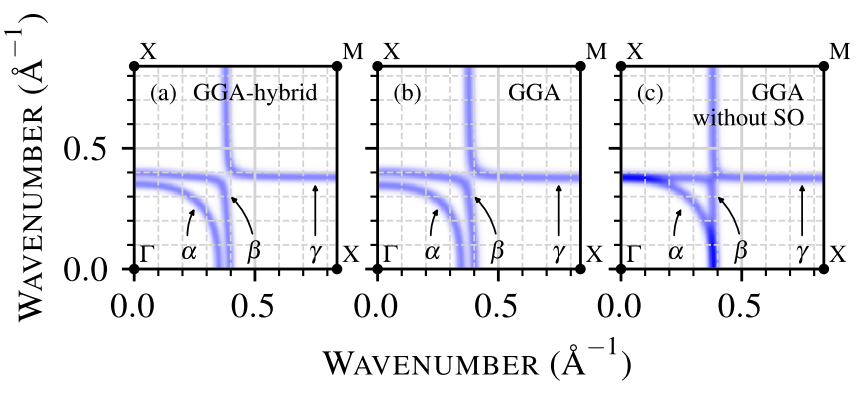

FIG. 6. Theoretical Fermi surface cut in the $\Gamma-\mathrm{X}-\mathrm{M}$ plane, calculated using (a) GGA hybrid, (b) GGA, and (c) GGA without spin-orbit interaction. 
taken as an indication that the Fermi surfaces are in essence determined by the Re $5 d t_{2 g}$.

Panels (b) and (c) of Fig. 6 compare the Fermi surface with and without spin-orbit coupling. The differences are quite dramatic. The $\alpha$ and $\beta$ sheets are separated when spin-orbit interaction is present, in the $\Gamma-\mathrm{X}$ direction there is a momentum splitting of approximately $0.05 \AA^{-1}(6 \% \overline{\Gamma X})$. In contrast, without spin-orbit interaction, these two sheets are degenerate in the $\Gamma$-X direction and are close to each other over large portions of the Brillouin zone. This demonstrates that the spin-orbit coupling is essential to explain the experimentally observed Fermi surface, see Fig. 5.

\section{SUMMARY}

We have performed angle-integrated and angle-resolved photoelectron spectroscopy measurements on $\mathrm{ReO}_{3}$. The data can be accurately explained by band structure calculations, provided that self-interaction effects are also taken into account. This affects mostly the position of the O $2 p$ bands relative to that of the $\operatorname{Re} 5 d$. Signatures of the $\operatorname{Re} 5 d$ spin-orbit interaction can be clearly observed in the dispersions of the bands as well as in the Fermi surface, which consists of the well-resolved $\alpha, \beta$, and $\gamma$ sheets.

\section{ACKNOWLEDGMENTS}

We thank Katharina Höfer and Christoph Becker for skillful technical assistance. The research in Dresden was partially supported by the DFG through SFB 1143 (Project No. 247310070). The experiments in Taiwan were facilitated by the Max Planck - POSTECH - Hsinchu Center for Complex Phase Materials.

\section{APPENDIX}

Figure 7 displays the $\mathrm{O} 1 s$ spectrum of $\mathrm{ReO}_{3}$ taken with different photon energies. The HAXPES spectrum at the bottom of the figure shows essentially a single sharp peak at about $529 \mathrm{eV}$ binding energy. The SXPES spectrum, on the other hand, reveals the presence of another peak (or set of peaks) at around $530 \mathrm{eV}$. The weight of this extra peak (or set of peaks) increases with lower photon energies.

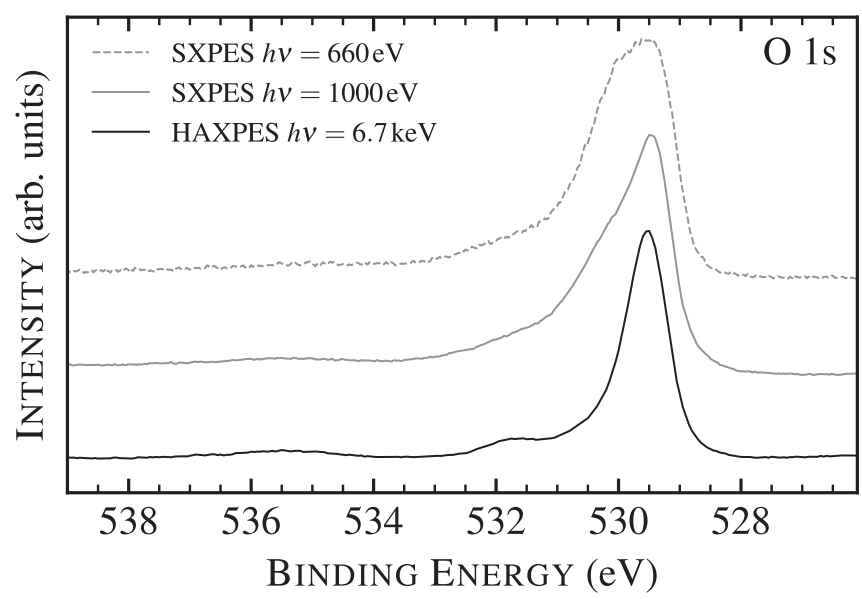

FIG. 7. O $1 s$ core-level photoemission spectra of $\mathrm{ReO}_{3}$ measured using, from bottom to top, HAXPES with $h v=6.7 \mathrm{keV}$ photons as well as SXPES with $h v=1000 \mathrm{eV}$ and $660 \mathrm{eV}$ photons.

To understand the photon energy dependence of the spectra, we recall that the inelastic mean free path of the outgoing photoelectrons is a function of their kinetic energy: it is of the order of 5-10 $\AA$ for $20-50 \mathrm{eV}$ and increases to about $70-100 \AA$ for $6-10 \mathrm{keV}$ [53], depending on the material composition. The kinetic energy of the $\mathrm{O} 1 s$ photoelectrons in Fig. 7 is close to $6 \mathrm{keV}$ in the HAXPES measurement (bottom curve), $470 \mathrm{eV}$ in the SXPES with $h v=1000 \mathrm{eV}$ (middle curve), and $130 \mathrm{eV}$ in the SXPES with $h v=660 \mathrm{eV}$ (top curve). The HAXPES spectrum thus probes more of the bulk electronic structure, while the SXPES is more sensitive to the surface region of the sample.

From this we can infer that the single-peak structure of the $\mathrm{O} 1 s$ in the HAXPES spectrum is representative of the bulk material, while the multipeak structure of the $\mathrm{O} 1 \mathrm{~s}$ in the SXPES indicates the presence of $\mathrm{O}$ species at the surface having electronic states that are different from those in the bulk. We can also infer from these experiments that we will need a kinetic energy of about $500 \mathrm{eV}$ or higher in order to obtain a clear bulk signal from $\mathrm{ReO}_{3}$. This in turn implies that we need $500 \mathrm{eV}$ or higher photon energies for the measurement of the valence band, both in the angle-integrated and the angle-resolved photoelectron spectroscopy experiments.
[1] N. Tsuda, K. Nasu, A. Yanase, and K. Siratori, Electronic Conduction in Oxides (Springer, Berlin, Heidelberg, 1991), pp. 105-286.

[2] M. Imada, A. Fujimori, and Y. Tokura, Rev. Mod. Phys. 70, 1039 (1998).

[3] S.-W. Cheong and M. Mostovoy, Nat. Mater. 6, 13 (2007).

[4] D. I. Khomskii, Transition Metal Compounds (Cambridge University Press, Cambridge, England, 2014).

[5] F. Zumsteg and T. Pearsall, Solid State Commun. 16, 751 (1975).

[6] A. Ferretti, D. Rogers, and J. Goodenough, J. Phys. Chem. Solids 26, 2007 (1965).
[7] C. King, H. Kirsch, and T. Geballe, Solid State Commun. 9, 907 (1971).

[8] T. P. Pearsall and C. A. Lee, Phys. Rev. B 10, 2190 (1974).

[9] T. Tanaka, T. Akahane, E. Bannai, S. Kawai, N. Tsuda, and Y. Ishizawa, J. Phys. C 9, 1235 (1976).

[10] P. B. Allen and W. W. Schulz, Phys. Rev. B 47, 14434 (1993).

[11] T. Chatterji, P. F. Henry, R. Mittal, and S. L. Chaplot, Phys. Rev. B 78, 134105 (2008).

[12] T. Chatterji, P. G. Freeman, M. Jimenez-Ruiz, R. Mittal, and S. L. Chaplot, Phys. Rev. B 79, 184302 (2009).

[13] E. E. Rodriguez, A. Llobet, T. Proffen, B. C. Melot, R. Seshadri, P. B. Littlewood, and A. K. Cheetham, J. Appl. Phys. 105, 114901 (2009). 
[14] Y. Liu, Z. Wang, M. Wu, Q. Sun, M. Chao, and Y. Jia, Comput. Mater. Sci. 107, 157 (2015).

[15] J. Purans, P. Fornasini, S. E. Ali, G. Dalba, A. Kuzmin, and F. Rocca, Phys. Rev. B 92, 014302 (2015).

[16] J.-E. Jørgensen, J. Staun Olsen, and L. Gerward, J. Appl. Crystallogr. 33, 279 (2000).

[17] T. Chatterji and G. McIntyre, Solid State Commun. 139, 12 (2006).

[18] S. Horiuchi, N. Kimizuka, and A. Yamamoto, Nature (London) 279, 226 (1979).

[19] S. Ling, D. Mei, and M. Gutowski, Catal. Today 165, 41 (2011).

[20] L. F. Mattheiss, Phys. Rev. 181, 987 (1969).

[21] L. F. Mattheiss, Phys. Rev. B 2, 3918 (1970).

[22] L. F. Mattheiss, Phys. Rev. B 6, 4718 (1972).

[23] H. W. Myron, R. P. Gupta, and S. H. Liu, Phys. Rev. B 8, 1292 (1973).

[24] M. G. Stachiotti, F. Corà, C. R. A. Catlow, and C. O. Rodriguez, Phys. Rev. B 55, 7508 (1997).

[25] F. Cora, M. Stachiotti, C. Catlow, and C. Rodriguez, J. Phys. Chem. B 101, 3945 (1997).

[26] J. Feinleib, W. J. Scouler, and A. Ferretti, Phys. Rev. 165, 765 (1968).

[27] J. H. Weaver and D. W. Lynch, Phys. Rev. B 6, 3620 (1972).

[28] S. Marcus, Phys. Lett. A 27, 584 (1968).

[29] F. S. Razavi and W. R. Datars, Can. J. Phys. 54, 845 (1976).

[30] G. K. Wertheim and S. Hüfner, Phys. Rev. Lett. 28, 1028 (1972).

[31] G. K. Wertheim, L. F. Mattheiss, M. Campagna, and T. P. Pearsall, Phys. Rev. Lett. 32, 997 (1974).

[32] R. Kasowski and W. Riggs, Phys. Lett. A 39, 387 (1972).

[33] A. Fujimori, F. Minami, T. Akahane, and N. Tsuda, J. Phys. Soc. Jpn. 49, 1820 (1980).

[34] O. Y. Khyzhun, Metallofiz. Noveishie Tekhnol. 27, 805 (2005).

[35] A. Fujimori, I. Hase, H. Namatame, Y. Fujishima, Y. Tokura, H. Eisaki, S. Uchida, K. Takegahara, and F. M. F. de Groot, Phys. Rev. Lett. 69, 1796 (1992).

[36] A. Fujimori, J. Phys. Chem. Solids 53, 1595 (1992), Special Issue, Elecronic Structure and Fermiology of High- $T_{\mathrm{c}}$ Superconductors.

[37] A. Fujimori, I. Hase, Y. Tokura, M. Abbate, F. de Groot, J. Fuggle, H. Eisaki, and S. Uchida, Phys. B: Condens. Matter 186-188, 981 (1993).

[38] H.-M. Tsai, H.-W. Fu, C.-Y. Kuo, L.-J. Huang, C.-S. Lee, C.-Y. Hua, K.-Y. Kao, H.-J. Lin, H.-S. Fung, S.-C. Chung, C.-F. Chang, A. Chainani, L. H. Tjeng, and C.-T. Chen, AIP Conf. Proc. 2054, 060047 (2019).

[39] J. Weinen, T. Koethe, C. Chang, S. Agrestini, D. Kasinathan, Y. Liao, H. Fujiwara, C. Schüßler-Langeheine, F. Strigari, T. Haupricht, G. Panaccione, F. Offi, G. Monaco, S. Huotari, K.-D. Tsuei, and L. Tjeng, J. Electron Spectrosc. Relat. Phenom. 198, 6 (2015).

[40] P. Blaha, K. Schwarz, G. K. Madsen, D. Kvasnicka, J. Luitz, R. Laskowsji, F. Tran, and L. Marks, WIEN2k, An Augmented Plane Wave + Local Orbitals Program for Calculating Crystal Properties (Karlheinz Schwarz, Techn. Universität Wien, Austria, 2018).
[41] P. Blaha, K. Schwarz, F. Tran, R. Laskowski, G. K. H. Madsen, and L. D. Marks, J. Chem. Phys. 152, 074101 (2020).

[42] J. P. Perdew, K. Burke, and M. Ernzerhof, Phys. Rev. Lett. 77, 3865 (1996).

[43] J. Heyd, G. E. Scuseria, and M. Ernzerhof, J. Chem. Phys. 118, 8207 (2003).

[44] J. Heyd and G. E. Scuseria, J. Chem. Phys. 121, 1187 (2004).

[45] F. Tran and P. Blaha, Phys. Rev. B 83, 235118 (2011).

[46] M. A. L. Marques, J. Vidal, M. J. T. Oliveira, L. Reining, and S. Botti, Phys. Rev. B 83, 035119 (2011).

[47] R. M. Martin, Electronic Structure: Basic Theory and Practical Methods, 2nd ed. (Cambridge University Press, Cambridge, England, 2020).

[48] M. Trzhaskovskaya, V. Nefedov, and V. Yarzhemsky, At. Data Nucl. Data Tables 77, 97 (2001).

[49] M. Trzhaskovskaya, V. Nefedov, and V. Yarzhemsky, At. Data Nucl. Data Tables 82, 257 (2002).

[50] M. Trzhaskovskaya, V. Nikulin, V. Nefedov, and V. Yarzhemsky, At. Data Nucl. Data Tables 92, 245 (2006).

[51] D. Takegami, L. Nicolaï, T. C. Koethe, D. Kasinathan, C. Y. Kuo, Y. F. Liao, K. D. Tsuei, G. Panaccione, F. Offi, G. Monaco, N. B. Brookes, J. Minár, and L. H. Tjeng, Phys. Rev. B 99, 165101 (2019).

[52] D. Takegami, D. Kasinathan, K. K. Wolff, S. G. Altendorf, C. F. Chang, K. Hoefer, A. Melendez-Sans, Y. Utsumi, F. Meneghin, T. D. Ha, C. H. Yen, K. Chen, C. Y. Kuo, Y. F. Liao, K. D. Tsuei, R. Morrow, S. Wurmehl, B. Büchner, B. E. Prasad, M. Jansen et al., Phys. Rev. B 102, 045119 (2020).

[53] C. J. Powell and A. Jablonski, J. Phys. Chem. Ref. Data 28, 19 (1999).

[54] P. W. Tasker, J. Phys. C 12, 4977 (1979).

[55] A. Fujimori, F. Minami, and N. Tsuda, Surf. Sci. 121, 199 (1982).

[56] A. Zangwill, Physics at Surfaces (Cambridge University Press, Cambridge, England, 1988).

[57] C. Noguera, Physics and Chemistry at Oxide Surfaces (Cambridge University Press, Cambridge, England, 1996).

[58] R. Hesper, L. H. Tjeng, A. Heeres, and G. A. Sawatzky, Phys. Rev. B 62, 16046 (2000).

[59] C. F. Chang, Z. Hu, S. Klein, X. H. Liu, R. Sutarto, A. Tanaka, J. C. Cezar, N. B. Brookes, H.-J. Lin, H. H. Hsieh, C. T. Chen, A. D. Rata, and L. H. Tjeng, Phys. Rev. X 6, 041011 (2016).

[60] J. Ghijsen, L. H. Tjeng, J. van Elp, H. Eskes, J. Westerink, G. A. Sawatzky, and M. T. Czyzyk, Phys. Rev. B 38, 11322 (1988).

[61] L. H. Tjeng, C. T. Chen, and S.-W. Cheong, Phys. Rev. B 45, 8205 (1992).

[62] L. H. Tjeng, M. B. J. Meinders, J. van Elp, J. Ghijsen, G. A. Sawatzky, and R. L. Johnson, Phys. Rev. B 41, 3190 (1990).

[63] A. K. Efimenko, N. Hollmann, K. Hoefer, J. Weinen, D. Takegami, K. K. Wolff, S. G. Altendorf, Z. Hu, A. D. Rata, A. C. Komarek, A. A. Nugroho, Y. F. Liao, K.-D. Tsuei, H. H. Hsieh, H.-J. Lin, C. T. Chen, L. H. Tjeng, and D. Kasinathan, Phys. Rev. B 96, 195112 (2017). 